\title{
Enhanced expression of Vastatin inhibits angiogenesis and prolongs survival in murine orthotopic glioblastoma model
}

\author{
Yi Li', Jun Li', Yat Ming Woo², Zan Shen ${ }^{3}$, Hong Yao ${ }^{4}$, Yijun Cai ${ }^{1}$, Marie Chia-mi Lin ${ }^{1}$ and Wai Sang Poon ${ }^{1 *}$
}

\begin{abstract}
Background: Antiangiogenic therapies are considered promising for the treatment of glioblastoma (GB). The noncollagenous C-terminal globular NC1 domain of type VIII collagen a1 chain, Vastatin, is an endogenous antiangiogenic polypeptide. Sustained enhanced expression of Vastatin was shown to inhibit tumour growth and metastasis in murine hepatocellular carcinoma models. In this study, we further explored the efficacy of Vastatin in the treatment of GB xenografts.
\end{abstract}

Method: Treatment of Vastatin was carried out using a nanopolymer gene vector PEl600-CyD-Folate (H1). Antiangiogenic effect of Vastatin was tested in vitro by using co-culture system and conditioned medium. An orthotopic GB murine model was established to examine the in vivo therapeutic effect of Vastatin alone treatment and its combination with temozolomide.

Results: Vastatin gene transfection mediated by H1 could target tumour cells specifically and suppress the proliferation of microvessel endothelial cells (MECs) through a paracrine inhibition manner. Enhancing Vastatin expression by intracerebral injection of $\mathrm{H1}$-Vastatin significantly prolonged animal survival from 48 to 75 days in GB murine model, which was comparable to the effect of Endostatin, the most studied endogenous antiangiogenic polypeptide. The diminished presence of CD34 positive cells in the GB xenografts suggested that Vastatin induced significant antiangiogenesis. Moreover, a synergistic effect in extending survival was detected when $\mathrm{H} 1$-Vastatin was administered with temozolomide (TMZ) in GB chemoresistant murine models.

Conclusion: Our results suggest, for the first time, that Vastatin is an antiangiogenic polypeptide with significant potential therapeutic benefit for GB. H1-Vastatin gene therapy may have important implications in re-sensitizing recurrent GB to standard chemotherapeutic agents.

Keywords: Vastatin, Glioblastoma, Antiangiogenesis, Gene therapy, Chemoresistance

\section{Background}

Glioblastoma (GB) is a lethal and aggressive human malignancy, accounting for over $60 \%$ of high-grade primary brain tumours $[1,2]$. In spite of significant technological advances in neurosurgery, anaesthesia, intensive care and oncology in the last few decades, GB remains incurable with a median overall survival of 15 months after its first diagnosis $[3,4]$. Antiangiogenesis is a therapeutic strategy

\footnotetext{
* Correspondence: wpoon@surgery.cuhk.edu.hk

'Brain Tumor Centre, Department of Surgery, The Chinese University of Hong Kong, Hong Kong, China
}

Full list of author information is available at the end of the article aiming at the suspension of tumour cells in a state of dormancy by disrupting their blood supply [5]. As hypervascularity, characterized by endothelial proliferation, is a hallmark of $\mathrm{GB}$, antiangiogenic therapies are naturally considered potential oncologic treatment options [6]. Studies focused on this therapeutic strategy have led to the development and approval of bevacizumab, a recombinant humanized monoclonal antibody against vascular endothelial growth factor (VEGF), for recurrent GB [7]. However, such clinical trials have produced inconsistent results and the overall benefits of bevacizumab on GB patients are being challenged [8-10]. Moreover, bevacizumab was not recommended for newly diagnosed GB due 
to its limited survival benefit and common adverse events $[11,12]$. Thus there is an urgent need to develop novel alternative antiangiogenic agents with more convincing therapeutic effects.

Vastatin is the C-terminal non-triple-helical (NC1) domain of the type VIII collagen $\alpha 1$ chain. It is an endogenous polypeptide that initially discovered to inhibit the proliferation and migration of bovine aortic endothelial cells [13]. Our recent study proved that Vastatin, which is normally expressed in normal liver tissue, was distinctly absent in hepatocellular carcinoma (HCC) and possessed antiangiogenic properties. Through interfering with proliferation and metabolism of endothelial cells, Vastatin inhibited tumour growth and prevented metastasis in HCC-bearing rats [14]. Concurrently a recombinant form of Vastatin, rhEDI-8 t, was discovered to be an angiogenesis inhibitor with potential therapeutic benefits for retinopathy-related neovascularization [15]. Since collagen VIII expression is known to be increased in brain tumours and participates in angiogenesis, we are interested in determining whether Vastatin could be used for the treatment of other hypervascular malignancies such as GB [16].

An ideal cancer therapeutic agent should be able to maintain predominantly high concentrations in the tumour thereby minimizing systemic adverse effects. We previously developed a polyplex-forming plasmid delivery agent, Folate-PEI600-CyD (H1). H1 formed nanoparticles with plasmid DNA and showed high affinity to cancer cells through binding to the folate receptors that enriched on cancer cell surface. It had high transfection efficiency especially on GB cells like U87 and U138 [17-19]. More importantly, $\mathrm{H} 1$ demonstrated low cytotoxicity and had little effect on normal cells. In the present study we aimed to test the feasibility of using $\mathrm{H} 1$ delivered Vastatin gene for treatment of GB xenografts. We report for the first time that enhancing Vastatin expression by $\mathrm{H} 1$ mediated gene transfection induced antiangiogenesis and prolonged survival of GB bearing mice, suggesting a promising treatment candidate for future GB drug development.

\section{Methods}

\section{Cell lines and Cell culture}

The murine tumour-derived microvessel endothelial cells (MECs) SVEC4-10EE2 and human GB cell lines U87MG were purchased from American Type Culture Collection (ATCC). They were maintained in either Minimum Essential Medium (MEM; Gibco) or Dulbecco's modified Eagle's medium (DMEM; Gibco) with $10 \%$ fetal-bovine-serum (FBS; Gibco) supplementation at $37{ }^{\circ} \mathrm{C}, 5 \% \mathrm{CO}_{2}$, and used for test within 20 passages after purchase.

GB cells with acquired TMZ resistance (ATR) were derived from U87MG cells through chronic exposure to TMZ. U87MG cells were first incubated in DMEM containing $20 \mu \mathrm{M}$ TMZ for 2 weeks, then subcultured into
DMEM with $200 \mu \mathrm{M}$ TMZ. Cells that managed to survive and proliferate in this medium for more than five passages were then collected. The final generated cells were considered resistant to TMZ treatment and named U87-ATR.

\section{Preparation of H1/DNA Polyplexes}

Plasmid pORF-EGFP, pORF-Endostatin and pORFVastatin were constructed by inserting DNA fragments encoding EGFP, Endostatin and Vastatin into the multiple cloning sites of the pORF-mcs expression vector (InvivoGen). The secretion of Vastatin and Endostatin protein were mediated by the Igk leader. The encoded gene was further confirmed by DNA sequencing.

The PEI600-CyD-Folate (H1) gene vector was synthesized as previously reported [18]. H1 polymer solution was added to pDNA solution in equal volumes to form the polyplexes. The ratio between the amount of nitrogen in PEI and the amount of phosphate in DNA (N/P ratio) was predetermined at 20 . The polyplex suspension was allowed to incubate at room temperature for $15 \mathrm{~min}$ before being used for transfection or injection.

\section{Orthotopic GB Murine Model}

Animal studies were performed in accordance with the protocol approved by the Animal Experimentation Ethics Committee of the Chinese University of Hong Kong (CUHK). Female nude mice, 6 to 8 weeks old, were purchased from the laboratory animal services center in CUHK. To establish the murine orthotopic GB model, animals were anaesthetised with ketamine:xylazine (100 mg/kg:10 mg/kg.body weight) and mounted into a stereotaxic frame (Stoelting Co.). A burr hole located $0.5 \mathrm{~mm}$ anterior to the coronal suture and $1.2 \mathrm{~mm}$ right to the sagittal suture was created. U87MG GB cells or U87-ATR cells were harvested and resuspended in phosphate buffered saline (PBS) to a concentration of $1 \times 10^{5}$ cells $/ \mu \mathrm{L}$. The needle of a Hamilton microsyringe was inserted through the burr hole to a depth of $2.5 \mathrm{~mm}$ where the right striatum is located. A total of $2 \times 10^{5}$ cells were slowly injected into this area at a rate of $0.2 \mu \mathrm{L} / \mathrm{min}$. The needle was slowly withdrawn $5 \mathrm{~min}$ after cell injection. The mice were then kept within far infrared lighting cabinets until recovery.

\section{Gene Expression Test}

Total 15 mice bearing U87MG xenografts were used for detection of gene expression after H1-Vastatin treatment. Treatments were performed by intracerebral injecting the H1-Vastatin polyplexes to the same location of tumour cell inoculation and ventricle nearby. A $20 \mu \mathrm{L}$ volume of $\mathrm{H} 1$-Vastatin solution was injected into each mouse at a rate of $0.5 \mu \mathrm{L} / \mathrm{min}$. This process was performed twice, on day 7 and day 14 post cell-inoculation, to achieve a total 
dosage of $20 \mu \mathrm{g}$ plasmid DNA. Mice were sacrificed on day 7 ( $1 \mathrm{~h}$ after the first treatment), 10, 14 ( $1 \mathrm{~h}$ after the second treatment), 17 and 21 , with 3 mice each time. The right hemispheres were isolated immediately for measurement of Vastatin mRNA level. Total RNA was extracted from brain tissues using TRIzol ${ }^{\circ}$ Reagent (Invitrogen) and then reverse transcribed to cDNA with SuperScript ${ }^{\circ}$ II Reverse Transcriptases (Invitrogen). The cDNA was then subjected to PCR assay and gel electrophoresis. The following primer sequences were used: Vastatin (forward:5'AAC TAC AAC CCG CAG ACA GG -3'; reverse:5'- TGA ATA GAG CAA CCC ACA CG -3'); Collagen VIII $\alpha 1$ (forward: 5' - ACT CTG TCA GAC TCA TTC AGG C -3'; reverse: 5'- CAA AGG CAT GTG AGG GAC TTG -3'); and GAPDH (forward:5'- GAA TCT ACT GGC GTC TTC ACC -3'; reverse:5'-GTC ATG AGC CCT TCC ACG ATG C - $\left.3^{\prime}\right)$.

\section{Animal survival tests}

Total 28 mice bearing U87MG xenografts were used to study the survival benefit of H1-Vastatin single treatment. On day 7 after model establishment, the mice were randomized into four groups, 7 mice for each group, and treated with H1-Vastatin, H1-Endostatin, H1-EGFP or PBS respectively. Treatments were performed using the same protocol for H1-Vastatin in gene expression test. The behaviors and survival of these mice were monitored daily. Mouse was sacrificed and recorded as dead when it lost over $20 \%$ of its body weight or exhibited serious behavioral disorders like seizures and limb weakness. The animal survivals after model establishment will be summarized in Kaplan-Meier survival curves.

To test the sensitivities of different model to TMZ treatment, 10 mice bearing U87MG xenografts and 10 mice bearing U87-ATR xenografts were used. On day 7 after model establishment, 5 mice with U87MG xenografts and 5 mice with U87-ATR xenografts were scheduled to be treated with TMZ, while the other 10 mice treated with PBS. TMZ was administered via intraperitoneal (i.p.) injection at a dose of $50 \mathrm{mg} / \mathrm{kg} /$ day. TMZ powder was first dissolved in dimethyl sulfoxide (DMSO; Sigma) and diluted with PBS before injection. This treatment was performed five times per week and lasted for 2 weeks. The behaviors and survival of animals were monitored daily as mentioned above.

To examine the combination effect of H1-Vastatin and TMZ, 20 mice bearing U87-ATR xenografts were used. On day 7 after model establishment, the mice were randomized into four groups, 5 mice for each group, and treated with H1-EGFP + PBS, H1-EGFP + TMZ, H1-Vastatin + PBS, or H1-Vastatin + TMZ respectively. Treatment of H1DNA and TMZ were performed using the same protocols mentioned above. The first TMZ administration was carried out $1 \mathrm{~h}$ after the first H1-DNA treatment on day 7 .
The behaviors and survival of animal were monitored and recorded daily.

\section{Histology study}

Nine mice bearing U87MG xenografts were used for histological study and microvessel density (MVD) analysis. On day 7 after model establishment, animals were divided into three groups, three mice in each group, and received the treatment of H1-Vastatin, H1-EGFP or PBS. All these mice were sacrificed on day 42 . Whole brain tissues were collected and processed through $10 \%$ formalin fixation and paraffin embedding. The tissue blocks were then cut at $5 \mu \mathrm{m}$ thickness with a microtome for histological analysis. Tumour structure assessment was performed using Hematoxylin \& Eosin (H\&E) staining. Angiogenesis in tumour tissues was detected by immunohistochemical staining using rabbit anti-CD34 primary antibody (Abcam) and HRP-linked anti-rabbit secondary antibody (Cell Signaling Technology), in accordance with a previous publication [20]. MVD was calculated by counting the percentage of CD34 positive cells in five randomly chosen high-power fields from each tumour.

\section{Cell proliferation assay}

For proliferation assays, $2 \times 10^{4}$ U87MG cells or $2 \times 10^{5}$ SVEC4-10EE2 MECs were seeded in a six-well plate and allowed to adhere. Twenty-four hours later, these cells were treated with $\mathrm{H} 1 /$ Vastatin or H1/EGFP $(\mathrm{N} / \mathrm{P}$ ratio $=20)$ for $6 \mathrm{~h}$ at a dosage of $10 \mu \mathrm{g}$ DNA per well and then incubated in DMEM with $10 \%$ FBS. Cell viability was assessed 2, 4, or 7 days later by trypan blue exclusion and viable cells were counted manually [21]. In the co-culture system, $2 \times 10^{5}$ MECs were seeded in a six-well plate while $2 \times 10^{5}$ U87MG cells were seeded onto the inner surface of the PET membrane located at the base of the Falcon ${ }^{\mathrm{TM}}$ culture insert (BD Biosciences). The insert was then placed into the sixwell plate where the MECs were seeded. H1/Vastatin or H1/EGFP treatment was added to the inner surface of the insert for $6 \mathrm{~h}$. Proliferation assays were carried out by counting the viable MECs at the same aforementioned time points.

To evaluate the inhibitory effects of secreted Vastatin on MEC proliferation, conditioned media were used. In brief, $2 \times 10^{6}$ U87MG or SVEC4-10EE2 cells were seeded in $100 \mathrm{~mm}$ culture dishes, treated with H1Vastatin or H1-EGFP at a dose of $10 \mu \mathrm{g}$ DNA per dish for $8 \mathrm{~h}$, then incubated in DMEM with 10\% FBS for $96 \mathrm{~h}$. The conditioned media were collected and centrifuged at $600 \mathrm{~g}, 4{ }^{\circ} \mathrm{C}$ for $10 \mathrm{~min}$. SVEC4-10EE2 MECs were seeded in a 96-well plate at a density of 5000 cells per well. After cell attachment, the media were changed to serial dilutions of conditioned media with 10\% FBS. Seven days later, 3-(4,5-dimethyl-2-thiazolyl)-2,5-diphenyl-2H-tetrazolium bromide (MTT) was added to the 
media and incubated for $2 \mathrm{~h}$. The media were then changed to dimethyl sulfoxide (DMSO) and assessed by colorimetric analysis at $570 \mathrm{~nm}$.

\section{In vitro temozolomide resistance testing}

For proliferation inhibition, 2000 U87MG or U87-ATR cells were seeded into each well of a 96-well plate and treated with increasing concentrations of TMZ. MTT assays were used to examine cell viability 4 days later. For clonogenic survival assays, U87MG or U87-ATR cells were seeded into a six-well plate at a density of 500 cells per well. The media were then changed to DMEM containing $10 \% \mathrm{FBS}$ and $100 \mu \mathrm{M}$ TMZ for incubation. On day 14 the number of colonies containing more than 50 cells were counted.

\section{Statistical analysis}

Mice survival was analysed with PASW Statistics Version 18 (SPSS Inc., Chicago, Illinois). Comparisons in proliferation tests and MVD analysis were conducted by one-way analysis of variance or two-tailed Student's t test. Comparisons of animal survivals were performed using Log-rank test. $P<0.05$ was considered statistically significant.

\section{Results}

H1-Vastatin transfected GB cells and inhibited MECs proliferation through paracrine suppression

The $\mathrm{H} 1$ gene vector was designed specifically to target tumour cells [18]. We proposed that $\mathrm{H} 1$ mediated gene transfection could restrict the expression and secretion of therapeutic agents in tumour areas and prevent systemic side effects. To prove this idea, GB U87MG cells and mouse MECs SVEC4-10EE2 were treated with either H1Vastatin or H1-EGFP in culture. Only U87MG cells treated by H1-Vastain showed enhanced Vastatin mRNA levels (Fig. 1a), which was consistent with previous report that H1-DNA nanoparticles transfected cancer cells specifically. We further conducted a series of proliferation tests on days 2, 4 and 7 after the cells received H1-Vastatin treatment. As anticipated, H1-Vastatin showed no significant influence on cell viability of either U87MG or MECs (Fig. 1b, left and middle). However in a U87MG and MECs trans-well coculture system, the proliferation of MECs was significantly inhibited on day 7 post-transfection (Fig. 1b, right; $P<0.05)$. This has demonstrated that the expression and secretion of Vastatin from H1-Vastatin transfected U87MG cells was necessary for inducing proliferation inhibition in MECs. Then we collected conditioned media (CM) from different culture groups on day 4 post-treatment. MECs SVEC4-10EE2 were seeded into a 96-well plate and incubated in serial dilutions of these CM. Proliferation test was performed 1 week later. Cell viabilities from different culture conditions were normalized to the NO CM culture group to generate an inhibition curve (Fig. 1c). The results showed that CM collected form H1-Vastatin treated U87MG reduced MECs proliferation in a dose-dependent way, while CM from H1-Vastatin treated MECs or H1EGFP treated U87MG had no such effect. This further supported our anticipation that H1-Vastatin could induce Vastatin secretion from tumour cells and suppress MECs proliferation by paracrine inhibition.

\section{Administration of $\mathrm{H} 1-$ Vastatin prolonged survival in GB- bearing mice}

The therapeutic benefit of H1-Vastatin was studied on GB-bearing mice and compared with PBS, EGFP and Endostatin. An orthotopic GB model was established by intracranial inoculation of U87MG cells into the nude mice. Intracerebral injections of $\mathrm{H} 1$-Vastatin, $\mathrm{H} 1$ Endostatin, H1-EGFP or PBS were performed on day 7 and day 14 after cells inoculation. Sustained expression enhancement of intracranial Vastatin level was observed after H1-Vastatin treatment (Fig. 2a). H1-Vastatin successfully prolonged animal survival from a median of 48 days (PBS treated group) to 75 days $(P<0.01, n=7$ for each group; Fig. 2b). The animal survival was also significantly extended in the Endostatin treated group (median survival of 64 days; $P<0.01$ against the PBS treated group). H1-EGFP caused no significant difference on animal survival (median survival of 51 days), suggesting that the vector per se did not interfere with the test. However, no significant difference in animal survival was detected between the Vastatin group and the Endostatin group. These results imply that Vastatin has a potent anti-tumour activity in this GB model, and is comparable to the well studied endogenous antiangiogenic agent Endostatin.

\section{Administration of H1-Vastatin decreased microvessel density (MVD) in GB-bearing mice}

For histological assessment, mice bearing GB xenografts were treated with PBS, H1-EGFP or H1-Vastatin respectively ( $n=3$ for each group). The animals were sacrificed at day 42 post tumour cell inoculation. The brain tissues were then fixed in formalin, embedded into paraffin blocks and processed for slicing and staining. Angiogenesis was detected by immunohistochemical staining against cells expressing CD34, a protein marker for blood vessel endothelial cells. Results showed that H1-Vastatin significantly reduced CD34+ cells in brain tumours (Fig. 3a). Microvessel density in the H1Vastatin treated group $(7.3 \pm 1.9)$ was significantly lower than those in the PBS $(13.7 \pm 1.8, P<0.05)$ and $\mathrm{H} 1$ EGFP $(14.5 \pm 2.9, \quad P<0.05)$ treated groups (Fig. 3b). These results indicated that Vastatin induced angiogenesis inhibition and eliminated tumor microvessels in the orthotopic GB model, which could be the underlying mechanism of its survival benefits. 
a

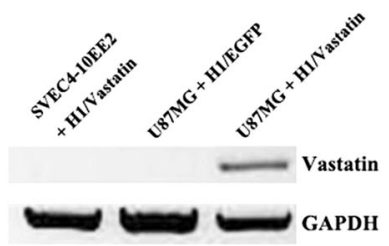

b

U87

SVEC4-10EE2

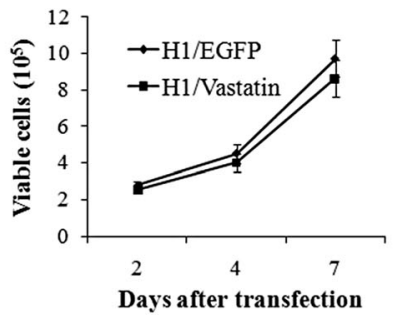

C
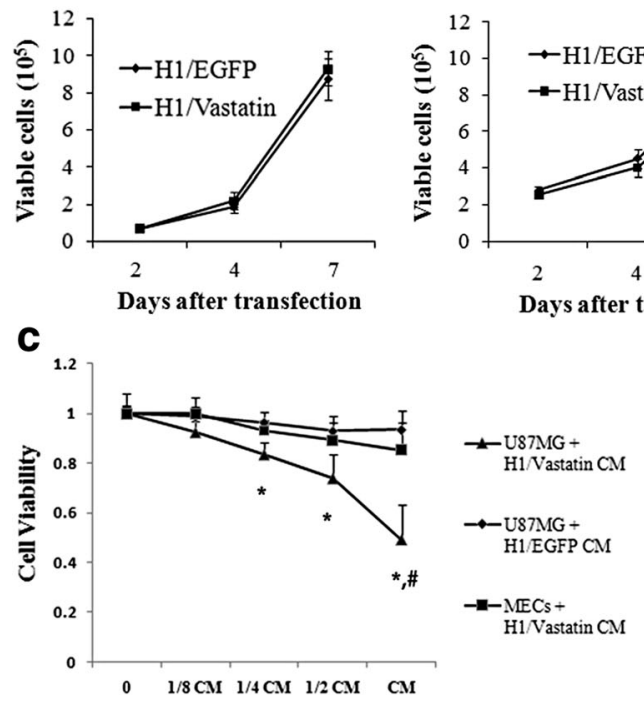

SVEC4-10EE2

(U87 coculture)

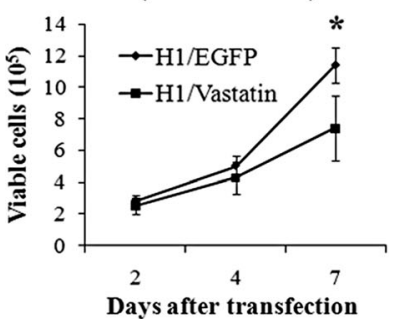

Fig. 1 H1-Vastatin transfectd tumour cells specifically and suppressed MECs proliferation through paracrine inhibition. a H1 mediated gene transfections targeted only the tumour cells. Enhanced transcription level of Vastatin was only detected in the H1-Vastatin treated U87MG cells. b Proliferation curves of cells treated by H1-Vastatin or H1-EGFP. H1-Vastatin did not affected the proliferation of U87MG cells or MECs in separate culture condition. In the U87MG and MECs co-culure system, H1-Vastatin significantly suppressed the MECs growth on day 7 post treatment $(P<0.05)$. c Inhibition curves showing effects of different conditioned media (CM) on MECs proliferation. Conditioned medium from H1-Vastatin treated U87MG cells significantly decreased the cell viability of MECs in a dosage dependant way ${ }^{*} P<0.05$ against CM from H1-EGFP treated U87MG cells; $P<0.05$ against CM from H1-Vastatin treated MECs), suggesting Vastatin secreted by tumour cells inhibited neovascularization in paracrine manner

\section{H1-Vastatin synergized with TMZ in chemoresistant GB model}

TMZ is an alkylating agent which damages tumor cell DNA and triggers cell death. It has been demonstrated to confer moderate survival benefits for GB patients. To test whether H1-Vastatin could facilitate current management of $\mathrm{GB}$, we performed a combined treatment of Vastatin and TMZ on GB orthotopic model. We also established a TMZ resistant GB model, since intrinsic and acquired chemoresistance are the main clinical challenges encountered in TMZ therapy. To induce a stable TMZ resistant cell line, U87MG cells were exposed to TMZ containing medium for a long term incubation. The generated cells, named U87-ATR, were confirmed to be TMZ resistant in both proliferation and survival assays (Fig. 4a). Nude mice intracranially inoculated with U87-ATR cells had significantly shorter survival (median survival of 25 days, $n=5$ ) than those with U87MG cells (median survival of 50 days; $P<0.05$ ). TMZ was found extremely effective in treating U87MG bearing mice, with all the animals in this group survived the total duration of 100 days. In contrast, mice with U87-ATR xenografts showed no significant response to TMZ treatment (median survival of 29 days; Fig. 4c). Our results further showed that H1-Vastatin was effective in the treatment of this TMZ resistant model, and significantly extended the median survival from 23 days of the H1-EGFP treated group to 34 days (Fig. $4 \mathrm{~d} ; n=5, P<0.05$ ). More interestingly, a synergistic effect was noted between $\mathrm{H} 1$ Vastatin and TMZ, which further prolonged the median survival to 54 days $(P<0.01$ against $\mathrm{H} 1$-EGFP treated group; $P<0.05$ against $\mathrm{H} 1$-Vastatin single treatment group).

\section{Discussion}

Angiogenesis is the physiological process by which new blood vessels develop from pre-existing vessels. In normal tissues, it is precisely regulated by a series of angiogenic stimulators and inhibitors. In the state of tumour growth, the balance between the stimulators and inhibitors is 


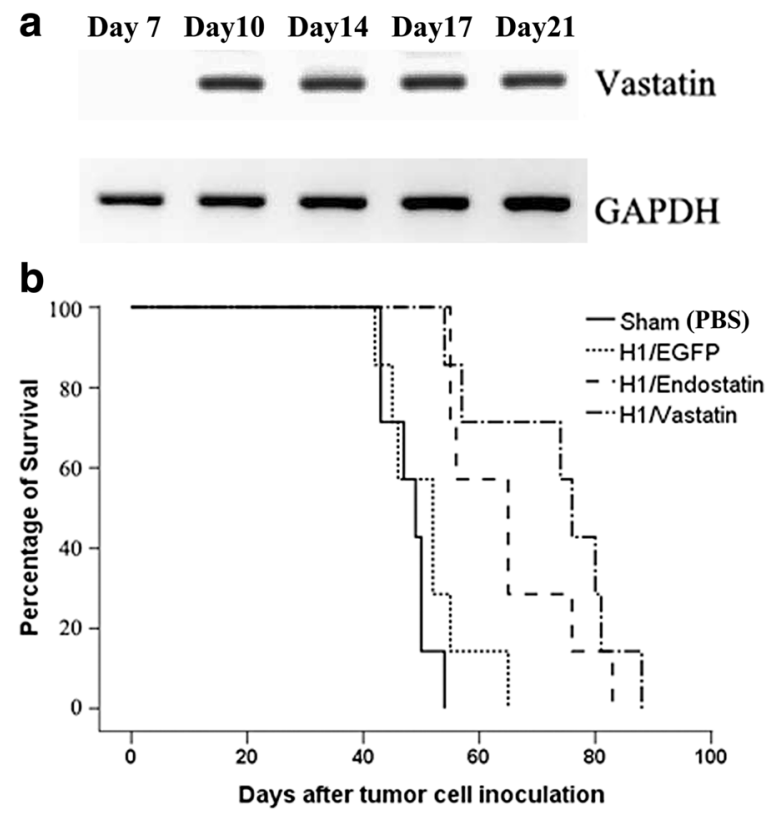

Fig. 2 Administration of H1-Vastatin increased intracranial Vastatin expression and significantly prolonged survival of GB bearing mice. a Bands of Vastatin exclusive PCR products in agarose gel electrophoresis. H1-Vastatin significantly enhanced the mRNA level of Vastatin in the right hemispheres of treated mice, which lasted over 2 weeks. b Survival curves of GB bearing mice $(n=7)$. H1-Vastatin and H1-Endostatin treatment significantly prolonged the median survival time of GB bearing mice to 75 and 64 days respectively, from 48 and 51 days for the PBS and H1-EGFP treated groups $(P<0.05)$. There was no significant difference in survival time between $\mathrm{H} 1$-Vastatin and $\mathrm{H} 1$-Endostatin treated groups

tipped, towards an "angiogenic switch" [22]. VEGF is one of these stimulators and plays a predominant role in regulating tumour angiogenesis. A humanized monoclonal antibody against VEGF, bevacizumab, has been shown to exhibit treatment response resulting in a longer progression-free survival in GB patients [23, 24]. However, angiogenic inhibitors like bevacizumab which target a single pathway often encounter rapid onset resistance through alternative pathways [25]. Studies that aimed to overcome this resistance have suggested the utilization of a combination of single-pathway targeted antiangiogenic agents [26]. Another alternative is to use broad-spectrum antiangiogenic agents. Endostatin, for example, is a 20kDA C-terminal cleavage fragment of collagen type XVIII and possesses the broadest anti-cancer spectrum. It targets angiogenesis regulatory genes that comprise of more than $12 \%$ of the human genome [27]. Approved by the State Food and Drug Administration of the People's Republic of China, Endostatin is currently a treatment option for nonsmall-cell lung cancer. Several reports also suggest that Endostatin might be effective in inhibiting tumour growth in malignant glioma in animal models [28-30].

Endostatin represents a group of endogenous angiogenic inhibitors that are fragments of larger extracellular matrix
(ECM) molecules. During angiogenesis, the breakdown of the ECM is a prerequisite for the initiation of sprouting. Endogenous antiangiogenic components are released during this process and act as focal natural feedback [15]. Among them are the NC1 domains cleaved from collagen molecules. Endostatin is the NC1 domain of collagen XVIII. Others include Arresten, Canstatin and Tumstatin from collagen IV, Restin from collagen XV 1 , and Vastatin from collagen VIII [13, 31-34]. They form a family collectively referred to as collagen-derived antiangiogenic factors (CDAFs). In cancer studies CDAFs have been reported to be effective in suppressing tumour progression, both in vitro and in vivo [35-37]. Furthermore, these endogenous inhibitors, having been demonstrated to be safe, acting on multiple proangiogenic pathways, are therefore attractive therapeutic candidates [38, 39].

Vastatin is a CDAF from type VIII collagen. Type VIII collagen is present in the ECM of sclera, skin and the renal glomerulus participating in their vascularization [40]. In contrast, Vastatin, contributes to the suppression of ocular neovascularization [15]. The potential of Vastatin in tumour treatment is not fully explored, even though type VIII collagen is highly expressed in selected solid tumours. As far as we know, we are the first to introduce Vastatin into preclinical malignant tumour studies. In our previous report, Vastatin is absent in human HCC, and rAAV-Vastatin infection effectively inhibites proliferation, migration and microvessel formation activities in MECs [14]. In this study we further demonstrate that Vastatin can inhibit angiogenesis and may be of therapeutic benefit in GB. Mechanism studies from our previous HCC research showed that Vastatin inhibited cellular metabolism, Notch and AP-1 signaling pathways [14]. Considering this result was from an in vitro study using MECs not specifically originated from HCC, we believed it could also be used for explaining the Vastatin induced antiangiogenesis in the GB model. The Notch signaling pathway in tumour angiogenesis is well-characterized. In general, delta-like ligand 4 (Dll4) interacts with Notch receptors and reduces VEGF signal transduction on stalk cells during sprouting, which contributes to the structural and functional integrity of newly formed vessels [41]. Inhibition of Dll4 and Notch signaling leads to functionally compromised vessels and suppresses tumour growth [42]. This may help to explain why Vastatin aggravated necrosis in our previous HCC study [14]. Changes in the degree of necrosis was not so obvious in current GB study, probably because the nature of the tumour inherently exhibits an abundance of necrosis as a hallmark feature. In GB, the Notch ligands provided by endothelial cells were also shown to be important for maintaining cancer stem-like cells (CSLCs) [43]. Inhibition of Notch signaling may cause growth inhibition of GSCs [44], which we believed was a possible mechanism underlying Vastatin's anti-glioma effect and 


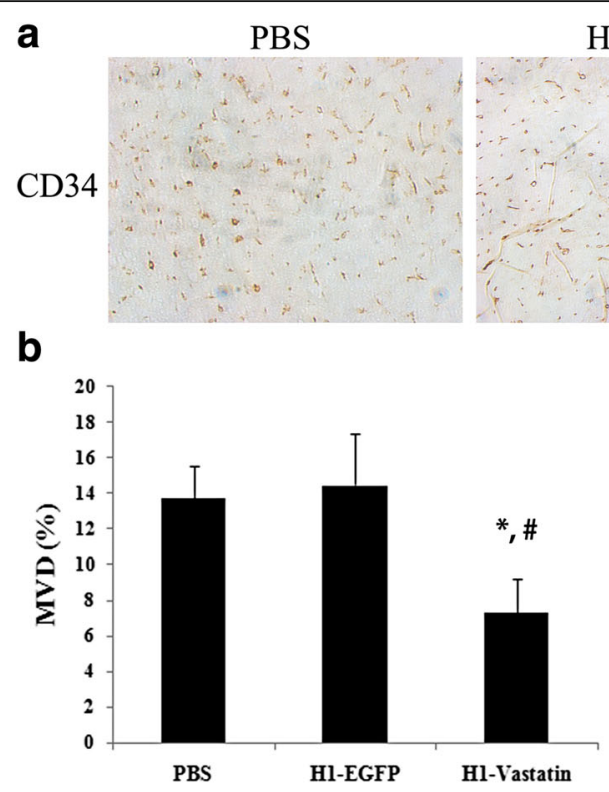

Fig. $3 \mathrm{H} 1$-Vastatin caused angiogenesis inhibition in vivo and decreased microvessel density. a Immunostaining of CD34, a vessel endothelia marker, on brain tumour sections. The CD34+ cell numbers were fewer in the H1-Vastatin treated group than the PBS and H1-EGFP treated groups. b Histogram showing the microvessel density (MVD) in tumours of different treatment groups. Percentage of microvessel endothelial cells in the $\mathrm{H1}$-Vastatin treated group was significant lower than in the PBS and H1-EGFP treated groups. $\left(n=3\right.$; ${ }^{*} P<0.05$ against PBS treated group, $\# P<0.05$ against H1-EGFP treated group)

distinguished Vastatin from traditional antiangiogenic agents. Unlike Notch signaling, the down-regulation of AP-1 and cell metabolism pathways seems to have a more direct influence on reducing MEC viability. AP-1 is a transcription factor that regulates a wide range of cellular processes, including cell growth, differentiation and apoptosis. In $\mathrm{GB}$, it mediates anoxia induced up-regulation of interleukin-8 (IL-8), a tumourigenic and proangiogenic chemokine [45]. In addition, AP-1 is involved in epidermal growth factor receptor (EGFR) mediated TMZ resistance [46]. Although we did not investigate the relationship between endothelium metabolism and antiangiogenic therapies, it was generally accepted that insufficient nutrients metabolism would lead to cell cycle arrest and apoptosis [47]. This is substantiated by evidence showing that enhanced glucose and glutamine metabolism in proliferating endothelial cells promotes tumour angiogenesis [48]. Altogether these findings depict a multi-targeted antiangiogenic pattern for Vastatin and considerably promotes its potential as an effective therapy for GB.

Safety is a primary concern in the treatment of brain tumours. Vastatin has been proven to be generally safe for systemic administration in previous HCC study [14]. However in this report, we highlighted the feasibility of recruiting $\mathrm{H} 1$ for local administration of antiangiogenic therapeutics. $\mathrm{H} 1$ induces endocytosis by binding to folate receptors that are highly expressed on certain tumour cell surfaces but not MECs [18]. Both the co-culture and conditioned medium test results imply that H1-Vastatin induced inhibition of MECs proliferation can be achieved by Vastatin secreted from adjacent GB tumour cells. In other words, H1-Vastatin selectively infects GB cells, restricting its antiangiogenic effects to the vicinity of the tumor thereby reducing the possibility of systemic adverse effects. Our observations that no deleterious effects were detected during the subsequent animal study is consistent with this hypothesis. This type of paracrine inhibition is also compatible with the "angiogenic switch" theory and restores the balance between angiogenic stimulators and inhibitors in the perivascular tumor microenvironment.

Whether antiangiogenic treatments could promote or attenuate chemotherapies is controversial, since changes in vascular integrity and permeability might complicate the passing of medications across the blood brain barrier. Clinical studies have combined bevacizumab with different cytotoxic chemotherapeutic agents in the treatment of either primary or recurrent GB. The results, unfortunately, were negative $[12,49,50]$. Nevertheless, the present study showed H1-Vastatin had a significant synergistic effect with TMZ in a chemoresistant GB murine model. This might be explained by the difference in anti-angiogenic mechanisms of bevacizumab and Vastatin, especially with regards to the Notch signaling regulation. Notch ligands expressed by endothelial cells are crucial for maintaining self-renewal of cancer stem cells [43]. Notch signaling pathway inhibition coupled with TMZ has been proven to exert an anti-glioma stem cell effect [51]. Moreover, the negative Notch-1 expression state was associated with 

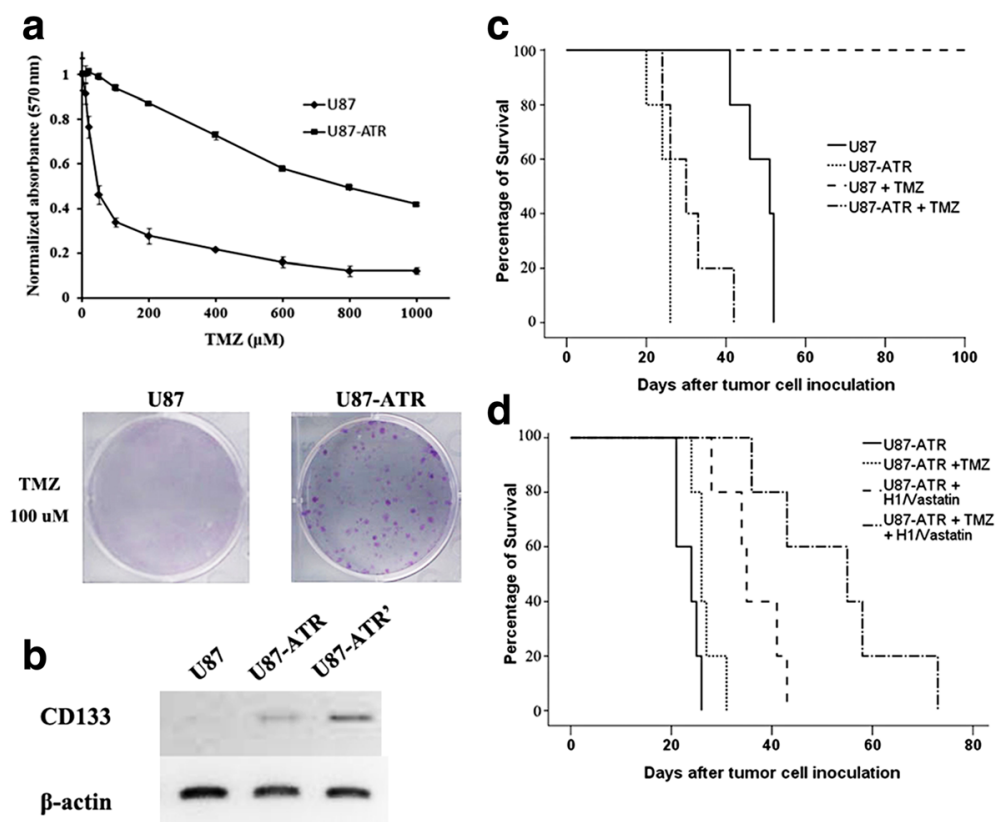

Fig. 4 Vastatin synergized with temozolomide in GB chemoresistant model. a TMZ resistance of U87-ATR cells. U87-ATR had a much higher half inhibited dosage of TMZ $(>800 \mu \mathrm{M})$ than U87MG $(<50 \mu \mathrm{M})$ in the proliferation test (upper). More U87-ATR than U87MG cells survived the treatment of $100 \mu \mathrm{M}$ TMZ and formed cell colonies (lower). b U87-ATR cells showed enhanced cancer stem cell property by expression of CSC marker CD133. U87-ATR' were cells amplified from a single cell clone which was picked out from TMZ treated U87-ATR. c Survival curves showing that GB model established using U87-ATR had a much shorter survival time ( 25 days) than using U87MG cells (50 days, $P<0.05$ ), and did not respond to TMZ treatment $(n=5)$. d Survival curves of TMZ resistance GB animals treated by H1-Vastatin and/or TMZ $(n=5)$. H1-Vastatin significantly prolonged the median survival of animals bearing U87-ATR xenografts to 34 days $(P<0.05)$. The combination of TMZ and H1-Vastatin showed even better therapeutic effects, with median survival extended to 54 days ( $P<0.01$ against $\mathrm{H1}$-EGFP treated group; $P<0.05$ against $\mathrm{H1}$-Vastatin single treatment group). This result suggested Vastatin synergized with TMZ and restored the sensitivity of chemoresistant mice to TMZ treatments

longer patient survival [52]. During the development of our mouse model, we introduced a group of cells with acquired TMZ resistance from original U87MG cells. These U87-ATR cells exhibited significant stem cell properties as evidenced by the high expression of cancer stem cell marker CD133 (Fig. 4b). The synergistic effect between Vastatin and TMZ in U87-ATR bearing mice might possibly be mediated by the suppression of Notch signaling in MECs, which subsequently lead to the eradication of perivascular niches for U87-ATR and other chemoresistant cancer stem like cells. However, it is one of our limitations that we did not show a direct inhibition effect of Vastatin treated MECs on U87-ATR cells, due to the lack of efficient cell-cell interaction model as well as the complications caused by the paracrine angiogenesis inhibition strategy. Studies to further investigate the synergistic effect between Vastatin and TMZ are ongoing, which we believe will help to discover the underlying mechanisms not just limited to a single pathway.

\section{Conclusion}

We report for the first time that Vastatin can induce antiangiogenesis and prolong survival in mice bearing GB orthotopic xenografts. We also confirm that H1-Vastatin offers a safe and efficient targeting method for GB antiangiogenic therapeutic tests. More importantly, a synergistic treatment effect is observed when Vastatin is coupled with TMZ therapy, which leads to the resensitization of initial chemoresistant GB model to TMZ treatment. At present, there is no effective treatment for patients with recurrent GB. Our results regarding the anti-tumor effects of Vastatin bear potential clinical therapeutic significance. The limitation of this study was that only used one animal model with one GB cell line were employed. Future studies should confirm these findings in models with more cell lines and different animals. Studies are also needed to further elucidate the pharmacological properties of Vastatin and its toxicological profile. In addition, combination effect of Vastatin and radiotherapy should be tested, since radiotherapy is a first-line treatment to GB patient and radioresistance is related to CSCs as well.

\section{Abbreviations}

AP-1: Activator protein-1; ATR: Acquired temozolomide resistance; CDAF: Collagen-derived antiangiogenic factor; CM: Conditioned medium; CSC: Cancer stem cell; ECM: Extracellular matrix; EGFP: Enhanced Green Fluorescent Protein; GB: Glioblastoma; HCC: Hepatocellular carcinoma; MEC: Microvessel endothelial cell; MVD: Microvessel density; PBS: Phosphate buffered saline; PEl: Polyethylenimine; rAAV: Recombinant adeno-associated virus; TMZ: Temozolomide; VEGF: Vascular endothelial growth factor 


\section{Acknowledgements}

We are grateful to Prof. Gong Chen and his team, Ms Jennifer Siu and Mr Johnny SZE for their technical assistance in $\mathrm{H} 1$ and plasmids preparation and cell culture.

\section{Funding}

This work was supported by the General Research Fund (CUHK 772910) from the Research Grants Council, Hong Kong. Otto Wong Brain Tumour Centre of the Chinese University of Hong Kong had partially supported a Research Assistant and laboratory consumables.

\section{Availability of data and materials}

The datasets during and/or analysed during the current study are available from the corresponding author on reasonable request.

\section{Authors' contributions}

YL designed this study, performed the experiments, analyzed data and prepared the manuscript; $J$ and $Y C$ designed this the study and participated in the cellular studies; HY and ZS participated in the nanoparticle preparation and animal treatment design; YW advised on clinical backgrounds and helped preparing this manuscript; ML and WP gave professional advices on study design and clinical backgrounds. All authors have read and approved the manuscript.

\section{Competing interest}

The authors declare that they have no competing interests.

\section{Consent for publication}

Not applicable.

\section{Ethics approval}

This research did not involve any human subjects. The manuscript does not contain any individual person's data. All the animal studies included in this manuscript were conducted according to the Regulations of Animal Welfare \& Ethics of Chinese University of Hong Kong, and has received ethics approval (Ref No. 10/100/MIS) from the Animal Experimentation Ethics Committee of the Chinese University of Hong Kong. The researchers who carried out this animal experiment were licensed by the local authority (Department of Health, HKSAR).

\section{Author details}

${ }^{1}$ Brain Tumor Centre, Department of Surgery, The Chinese University of Hong Kong, Hong Kong, China. ${ }^{2}$ Department of Neurosurgery, Kwong Wah Hospital, Hong Kong, China. ${ }^{3}$ Department of Oncology, Affiliated 6th People's Hospital, Shanghai Jiaotong University, Shanghai, China. ${ }^{4}$ Jiangsu Eng. Lab of Cancer Biotherapy, Xuzhou Medical College, Xuzhou, China.

\section{Received: 5 September 2016 Accepted: 8 February 2017} Published online: 13 February 2017

\section{References}

1. Dolecek TA, Propp JM, Stroup NE, Kruchko C. CBTRUS statistical report: primary brain and central nervous system tumors diagnosed in the United States in 2005-2009. Neuro Oncol. 2012;14 Suppl 5:v1-49.

2. Wen PY, Kesari S. Malignant gliomas in adults. N Engl J Med. 2008;359(5):492-507.

3. Van Meir EG, Hadjipanayis CG, Norden AD, Shu HK, Wen PY, Olson JJ. Exciting new advances in neuro-oncology: the avenue to a cure for malignant glioma. CA Cancer J Clin. 2010;60(3):166-93.

4. Johnson DR, Leeper HE, Uhm JH. Glioblastoma survival in the United States improved after Food and Drug Administration approval of bevacizumab: a population-based analysis. Cancer. 2013;119(19):3489-95.

5. Folkman J. Tumor angiogenesis: therapeutic implications. N Engl J Med. 1971;285(21):1182-6.

6. Gilbert MR. Antiangiogenic therapy for glioblastoma: complex biology and complicated results. J Clin Oncol. 2016;34(14):1567-9.

7. Norden AD, Young GS, Setayesh K, Muzikansky A, Klufas R, Ross GL, Ciampa AS, Ebbeling LG, Levy B, Drappatz J, et al. Bevacizumab for recurrent malignant gliomas: efficacy, toxicity, and patterns of recurrence. Neurology. 2008;70(10):779-87.

8. Pope WB, Xia Q, Paton VE, Das A, Hambleton J, Kim HJ, Huo J, Brown MS, Goldin J, Cloughesy T. Patterns of progression in patients with recurrent glioblastoma treated with bevacizumab. Neurology. 2011;76(5):432-7.
9. Junck L. Bevacizumab antiangiogenic therapy for glioblastoma. Neurology. 2011;76(5):414-5.

10. Wick W, Weller M, van den Bent M, Stupp R. Bevacizumab and recurrent malignant gliomas: a European perspective. J Clin Oncol. 2010;28(12):e188-189. author reply e190-182.

11. Gilbert MR, Dignam JJ, Armstrong TS, Wefel JS, Blumenthal DT, Vogelbaum MA, Colman H, Chakravarti A, Pugh S, Won M, et al. A randomized trial of bevacizumab for newly diagnosed glioblastoma. $\mathrm{N}$ Engl J Med. 2014;370(8):699-708.

12. Chinot OL, Wick W, Mason W, Henriksson R, Saran F, Nishikawa R, Carpentier AF, Hoang-Xuan K, Kavan P, Cernea D, et al. Bevacizumab plus radiotherapytemozolomide for newly diagnosed glioblastoma. N Engl J Med. 2014; 370(8):709-22.

13. Xu R, Yao ZY, Xin L, Zhang Q, Li TP, Gan RB. NC1 domain of human type VIII collagen (alpha 1) inhibits bovine aortic endothelial cell proliferation and causes cell apoptosis. Biochem Biophys Res Commun. 2001:289(1):264-8.

14. Shen Z, Yao C, Wang Z, Yue L, Fang Z, Yao H, Lin F, Zhao H, Sun YJ, Bian $X W$, et al. Vastatin, an Endogenous Antiangiogenesis Polypeptide That Is Lost in Hepatocellular Carcinoma, Effectively Inhibits Tumor Metastasis. Mol Ther. 2016:24(8):1358-68.

15. Zhang L, Shen X, Lu Q, Zhou Q, Gu J, Gan R, Zhang H, Sun X, Xie B. A potential therapeutic strategy for inhibition of ocular neovascularization with a new endogenous protein: rhEDI-8t. Graefe's Arch Clin Exp Ophthalmology. 2012;250(5):731-9.

16. Paulus W, Sage EH, Liszka U, Iruela-Arispe ML, Jellinger K. Increased levels of type VIII collagen in human brain tumours compared to normal brain tissue and non-neoplastic cerebral disorders. Br J Cancer. 1991:63(3):367-71.

17. Yao H, Ng SS, Huo LF, Chow BK, Shen Z, Yang M, Sze J, Ko O, Li M, Yue A, et al. Effective melanoma immunotherapy with interleukin-2 delivered by a novel polymeric nanoparticle. Mol Cancer Ther. 2011;10(6):1082-92.

18. Yao H, Ng SS, Tucker WO, Tsang YK, Man K, Wang XM, Chow BK, Kung HF, Tang GP, Lin MC. The gene transfection efficiency of a folate-PEl600cyclodextrin nanopolymer. Biomaterials. 2009;30(29):5793-803.

19. Hu BG, Liu LP, Chen GG, Ye CG, Leung KK, Ho RL, Lin MC, Lai PB. Therapeutic efficacy of improved alpha-fetoprotein promoter-mediated tBid delivered by folate-PEl600-cyclodextrin nanopolymer vector in hepatocellular carcinoma. Exp Cell Res. 2014;324(2):183-91.

20. Liu XM, Zhang QP, Mu YG, Zhang XH, Sai K, Pang JC, Ng HK, Chen ZP. Clinical significance of vasculogenic mimicry in human gliomas. J Neurooncol. 2011;105(2):173-9.

21. Shen Z, Yang ZF, Gao Y, Li JC, Chen HX, Liu CC, Poon RT, Fan ST, Luk JM, Sze $\mathrm{KH}$, et al. The kringle 1 domain of hepatocyte growth factor has antiangiogenic and antitumor cell effects on hepatocellular carcinoma. Cancer Res. 2008:68(2):404-14.

22. Ribatti D. Endogenous inhibitors of angiogenesis: a historical review. Leuk Res. 2009:33(5):638-44.

23. Cloughesy T. FDA accelerated approval benefits glioblastoma. Lancet Oncol. 2010;11(12):1120.

24. Kreisl TN, Kim L, Moore K, Duic P, Royce C, Stroud I, Garren N, Mackey M, Butman JA, Camphausen K, et al. Phase II trial of single-agent bevacizumab followed by bevacizumab plus irinotecan at tumor progression in recurrent glioblastoma. J Clin Oncol. 2009;27(5):740-5.

25. Norden AD, Drappatz J, Wen PY. Antiangiogenic therapies for high-grade glioma. Nat Rev Neurol. 2009:5(11):610-20.

26. Moreno Garcia V, Basu B, Molife LR, Kaye SB. Combining antiangiogenics to overcome resistance: rationale and clinical experience. Clin Cancer Res. 2012;18(14):3750-61.

27. Abdollahi A, Hahnfeldt P, Maercker C, Grone HJ, Debus J, Ansorge W, Folkman J, Hlatky L, Huber PE. Endostatin's antiangiogenic signaling network. Mol Cell. 2004;13(5):649-63.

28. Read TA, Sorensen DR, Mahesparan R, Enger PO, Timpl R, Olsen BR, Hjelstuen $\mathrm{MH}$, Haraldseth $\mathrm{O}$, Bjerkvig R. Local endostatin treatment of gliomas administered by microencapsulated producer cells. Nat Biotechnol. 2001;19(1):29-34.

29. Peroulis I, Jonas N, Saleh M. Antiangiogenic activity of endostatin inhibits C6 glioma growth. Int J Cancer. 2002;97(6):839-45.

30. Schmidt NO, Ziu M, Carrabba G, Giussani C, Bello L, Sun Y, Schmidt K, Albert M, Black PM, Carroll RS. Antiangiogenic therapy by local intracerebral microinfusion improves treatment efficiency and survival in an orthotopic human glioblastoma model. Clin Cancer Res. 2004;10(4):1255-62. 
31. Colorado PC, Torre A, Kamphaus G, Maeshima Y, Hopfer H, Takahashi K, Volk R, Zamborsky ED, Herman S, Sarkar PK, et al. Anti-angiogenic cues from vascular basement membrane collagen. Cancer Res. 2000;60(9):2520-6.

32. Kamphaus GD, Colorado PC, Panka DJ, Hopfer H, Ramchandran R, Torre A, Maeshima Y, Mier JW, Sukhatme VP, Kalluri R. Canstatin, a novel matrixderived inhibitor of angiogenesis and tumor growth. J Biol Chem. 2000; 275(2):1209-15.

33. Maeshima Y, Colorado PC, Kalluri R. Two RGD-independent alpha vbeta 3 integrin binding sites on tumstatin regulate distinct anti-tumor properties. J Biol Chem. 2000;275(31):23745-50.

34. John H, Radtke K, Standker L, Forssmann WG. Identification and characterization of novel endogenous proteolytic forms of the human angiogenesis inhibitors restin and endostatin. Biochim Biophys Acta. 2005;1747(2):161-70.

35. Monboisse JC, Oudart JB, Ramont L, Brassart-Pasco S, Maquart FX. Matrikines from basement membrane collagens: a new anti-cancer strategy. Biochim Biophys Acta. 2014;1840(8):2589-98.

36. Oliveira-Ferrer L, Wellbrock J, Bartsch U, Penas EM, Hauschild J, Klokow M, Bokemeyer C, Fiedler W, Schuch G. Combination therapy targeting integrins reduces glioblastoma tumor growth through antiangiogenic and direct antitumor activity and leads to activation of the pro-proliferative prolactin pathway. Mol Cancer. 2013;12(1):144.

37. Thevenard J, Ramont L, Mir LM, Dupont-Deshorgue A, Maquart FX, Monboisse JC, Brassart-Pasco S. A new anti-tumor strategy based on in vivo tumstatin overexpression after plasmid electrotransfer in muscle. Biochem Biophys Res Commun. 2013;432(4):549-52.

38. Chen Z, Guo W, Cao J, Lv F, Zhang W, Qiu L, Li W, Ji D, Zhang S, Xia Z, et al. Endostar in combination with modified FOLFOX6 as an initial therapy in advanced colorectal cancer patients: a phase I clinical trial. Cancer Chemother Pharmacol. 2015;75(3):547-57.

39. Herbst RS, Hess KR, Tran HT, Tseng JE, Mullani NA, Charnsangavej C, Madden T, Davis DW, McConkey DJ, O'Reilly MS, et al. Phase I study of recombinant human endostatin in patients with advanced solid tumors. J Clin Oncol. 2002;20(18):3792-803.

40. Shuttleworth CA. Type VIII collagen. Int J Biochem Cell Biol. 1997;29(10):1145-8.

41. Phng LK, Gerhardt H. Angiogenesis: a team effort coordinated by notch. Dev Cell. 2009:16(2):196-208.

42. Noguera-Troise I, Daly C, Papadopoulos NJ, Coetzee S, Boland P, Gale NW, Lin HC, Yancopoulos GD, Thurston G. Blockade of Dll4 inhibits tumour growth by promoting non-productive angiogenesis. Nature. 2006;444(7122): 1032-7.

43. Zhu TS, Costello MA, Talsma CE, Flack CG, Crowley JG, Hamm LL, He X, Hervey-Jumper SL, Heth JA, Muraszko KM, et al. Endothelial cells create a stem cell niche in glioblastoma by providing NOTCH ligands that nurture self-renewal of cancer stem-like cells. Cancer Res. 2011;71(18): $6061-72$.

44. Saito N, Fu J, Zheng S, Yao J, Wang S, Liu DD, Yuan Y, Sulman EP, Lang FF, Colman $\mathrm{H}$, et al. A high Notch pathway activation predicts response to gamma secretase inhibitors in proneural subtype of glioma tumor-initiating cells. Stem Cells. 2014;32(1):301-12.

45. Brat DJ, Bellail AC, Van Meir EG. The role of interleukin-8 and its receptors in gliomagenesis and tumoral angiogenesis. Neuro Oncol. 2005;7(2):122-33.

46. Munoz JL, Rodriguez-Cruz V, Greco SJ, Ramkissoon SH, Ligon KL, Rameshwar P. Temozolomide resistance in glioblastoma cells occurs partly through epidermal growth factor receptor-mediated induction of connexin 43. Cell Death Dis. 2014;5:e1145.

47. Mason EF, Rathmell JC. Cell metabolism: an essential link between cell growth and apoptosis. Biochim Biophys Acta. 2011;1813(4):645-54.

48. Polet F, Feron O. Endothelial cell metabolism and tumour angiogenesis: glucose and glutamine as essential fuels and lactate as the driving force. J Intern Med. 2013;273(2):156-65.

49. Lai A, Tran A, Nghiemphu PL, Pope WB, Solis OE, Selch M, Filka E, Yong WH, Mischel PS, Liau LM, et al. Phase II study of bevacizumab plus temozolomide during and after radiation therapy for patients with newly diagnosed glioblastoma multiforme. J Clin Oncol. 2011;29(2):142-8.

50. Hasselbalch B, Lassen U, Hansen S, Holmberg M, Sorensen M, Kosteljanetz M, Broholm H, Stockhausen MT, Poulsen HS. Cetuximab, bevacizumab, and irinotecan for patients with primary glioblastoma and progression after radiation therapy and temozolomide: a phase II trial. Neuro Oncol. 2010; 12(5):508-16.
51. Yahyanejad S, King H, Iglesias VS, Granton PV, Barbeau LM, van Hoof SJ, Groot AJ, Habets R, Prickaerts J, Chalmers AJ, et al. NOTCH blockade combined with radiation therapy and temozolomide prolongs survival of orthotopic glioblastoma. Oncotarget. 2016;7(27):41251-64.

52. Saito N, Aoki K, Hirai N, Fujita S, Iwama J, Hiramoto Y, Ishii M, Sato K, Nakayama H, Harashina J, et al. Effect of Notch expression in glioma stem cells on therapeutic response to chemo-radiotherapy in recurrent glioblastoma. Brain Tumor Pathol. 2015;32(3):176-83.

\section{Submit your next manuscript to BioMed Central and we will help you at every step:}

- We accept pre-submission inquiries

- Our selector tool helps you to find the most relevant journal

- We provide round the clock customer support

- Convenient online submission

- Thorough peer review

- Inclusion in PubMed and all major indexing services

- Maximum visibility for your research

Submit your manuscript at www.biomedcentral.com/submit 